\title{
Cinética Química e Dinâmica Epidemiológica
} Mário Berberan e Santos

\begin{abstract}
Chemical Kinetics and Epidemiological Dynamics. Chemical Kinetics and Epidemiology are both devoted to the evolution of populations and have common aspects. It is shown that the SIR Kermack-McKendrick model of epidemic dynamics is mathematically identical to the autocatalysis with inhibition reaction mechanism. Autocatalysis with no inhibition, which has analytical solution (the logistic curve) and is by itself relevant in population dynamics, is a pedagogically sound way of introducing the problem. After establishing the connection between kinetics and epidemic dynamics, the SIR model is discussed and applied to an epidemic case study used in textbooks, and which is used to build several scenarios of current interest. The last part of the article is devoted to the application of some results of the model to the current COVID-19 pandemic, using data from Portugal, Italy and USA.
\end{abstract}

Tanto a Cinética Química como a Dinâmica Epidemiológica descrevem e interpretam a evolução de populações, tendo, por essa razão, muitos aspetos em comum. Neste artigo, estabelece-se o paralelo entre o modelo epidemiológico SIR de Kermack-McKendricke a autocatálise com inibição. A autocatálise sem inibição, que possui solução analítica (a curva logística), já por si relevante em dinâmica populacional, é uma boa forma de introduzir o problema. Após estabelecer a ligação entre Cinética Química e Dinâmica Epidemiológica, apresenta-se e discute-se em pormenor o modelo SIR, que é aplicado a um caso real de epidemia viral, dado como exemplo em livros de texto, e que é usado para construir vários cenários idealizados com relevância atual. Conclui-se o artigo com a aplicação de alguns resultados do modelo à pandemia de COVID-19, usando dados de Portugal, Itália e EUA.

\section{Autocatálise e equação logística}

Considere-se a reação elementar autocatalítica $[1,2]$

$S+I \stackrel{k}{\longrightarrow} 2 I$

em que S é o substrato e I o produto que catalisa a sua própria produção (definição de autocatálise). Tem de haver uma quantidade inicial não nula de produto para que a reação se possa dar. 0 mecanismo da reação pode ser representado pelo Esquema 1.

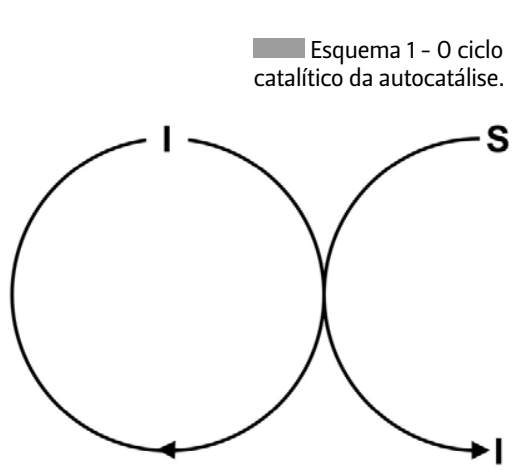


Um exemplo bem conhecido de uma reação deste tipo é a conversão do tripsinogénio em tripsina (uma protease), que esta própria catalisa, e que se dá no nosso organismo durante a digestão [3].

As equações de velocidade correspondentes à eq. 1 (autocatálise quadrática) são:

$$
\begin{aligned}
& \frac{d S}{d t}=-k S I \\
& \frac{d I}{d t}=k S I
\end{aligned}
$$

em que, por simplicidade, se omitem os parênteses retos, ou seja, Se I simbolizam as concentrações [S] e [I], respetivamente. A constante de velocidade ké bimolecular. As concentrações iniciais são $S_{0}$ e $I_{0}$, tendo-se $S_{0}$ $+I_{0}=S+I$. Sendo a reação irreversível, todo o $S$ acaba por ser convertido em $I$, tendo-se assim $I_{\infty}=S_{0}+I_{0}$. 0 número de moléculas de I produzidas por cada molécula de I, por unidade de tempo (frequência de conversão), é igual a $k S$.

A integração da eq. 3 é imediata,

$$
I=I_{0} \frac{I_{\infty}}{I_{0}+S_{0} \exp \left(-k I_{\infty} t\right)}
$$

Esta função do tempo é designada por curva logística $[4,5]$ (veja-se a Figura 1). Aplica-se à evolução de algumas populações de seres vivos, cujo aumento depende de alimentos, nutrientes ou outro elemento crítico, representado por S (inicialmente $S_{0}$ ) [5]. A mesma função foi usada na descrição do crescimento de organismos individuais [6]. Em todos estes casos, $k$ traduz um potencial intrínseco de crescimento. A curva logística também foi utilizada em estudos demográficos pioneiros, designadamente para representar a evolução da população dos EUA entre 1790 e 1910 [7].

Segue-se da eq. 4 que o crescimento é inicialmente exponencial. De facto, para tempos curtos e para $S_{0}>I_{0}$, a eq. 4 reduz-se a

$$
I=I_{0} \exp \left(k S_{0} t\right)
$$

Tanto a população (ou concentração) / como a velocidade de crescimento $\mathrm{d} / \mathrm{d} t$ duplicam a cada

$$
T_{2}=\frac{\ln 2}{k S_{0}}
$$

que é, pois, apenas determinado pela frequência característica [8]. Contudo, como S vai diminuindo, a velocidade de crescimento passa por um máximo $\left(d / / d t\right.$ ) no instante $t_{1 / 2}$ (que é o ponto de inflexão para I) e diminui a partir daí, como se mostra na Figura 1. A eq. 4 pode ser reescrita como

$$
I=\frac{I_{\infty}}{1+\exp \left[-k I_{\infty}\left(t-t_{1 / 2}\right)\right]}
$$

sendo $t_{1 / 2}$ o tempo necessário para a população atingir metade do valor máximo,

$$
t_{1 / 2}=\frac{\ln \left(S_{0} / I_{0}\right)}{k I_{\infty}}
$$

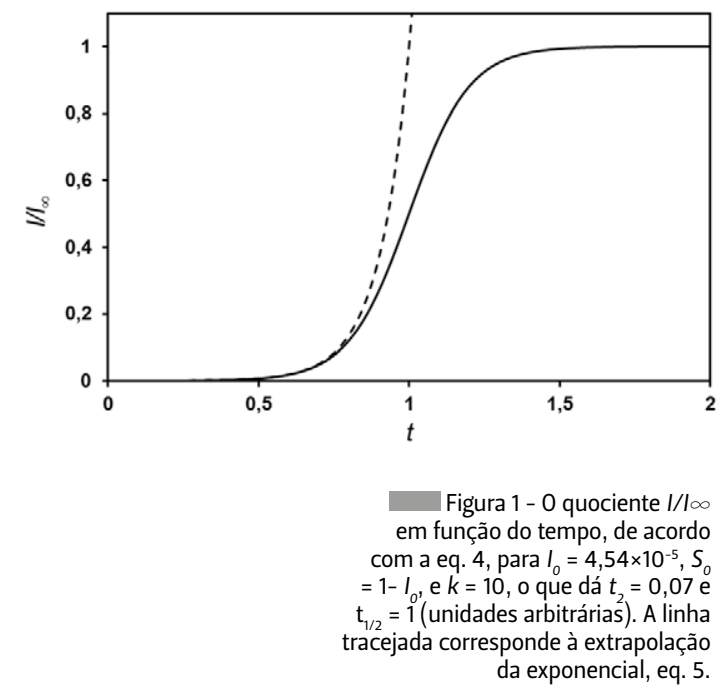

Resulta da eq. 8 que o tempo é tanto mais longo quanto menor for o valor inicial de I, I (aumentando o tempo de indução) ou mais elevado o valor inicial de $\mathrm{S}, \mathrm{S}_{0}$ (mais substrato disponível para o crescimento). 0 tempo de duplicação diminui com o aumento da constante de velocidade.

É de referir que o crescimento de culturas de bactérias (I), que inicialmente se pensava seguir a curva logística [9], é afinal melhor descrito pelo mecanismo de Monod [10], que tem em conta a "saturação" a partir de um certo nível de substrato,

$$
S+I \rightleftarrows(S I) \rightarrow 2 I
$$


0 mesmo se aplica a reações autocatalíticas, como a já referida conversão tripsinogénio-tripsina [3]. Ignorando este ponto, não essencial para a presente discussão, podemos perguntar o que sucederia se existisse uma reação competitiva que levasse à desativação irreversível do catalisador I. Tanto poderia ser uma reação unimolecular elementar, como uma reação com outro composto em excesso (por exemplo um inibidor), em ambos os casos:

$$
I \longrightarrow R
$$

sendo $R$ um certo produto, Esquema 2. pelos seus nomes: modelo SIR de Kermack-McKendrick [12-14], pela razão que se verá já de seguida. Neste modelo, a população é dividida em três categorias: Suscetíveis (S), que podem contrair a doença; Infetantes (ou Infeciosos), I, que podem transmitir a doença aos suscetíveis; e Removidos, $R$, que são infetantes entretanto curados e imunizados, ou falecidos que sucumbiram à doença (continuam a ser contabilizados para que a população se mantenha constante). Para além da imunização, admite-se no modelo que o período de latência é desprezável, ou seja, um indivíduo fica contagioso, assim que é infetado. De outra forma, é necessário considerar uma quarta categoria, os Expostos (infetados, mas ainda não contagiosos), e o modelo passa a ser o SEIR, Esquema 3.

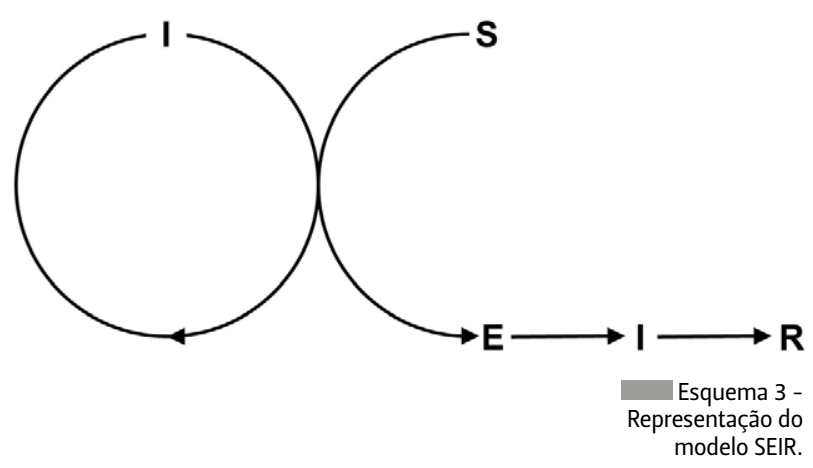

O modelo também pressupõe uma mistura perfeita de suscetíveis e infetantes, em resultado de completa liberdade de movimento no espaço do sistema (correspondente, em termos químicos, a uma reação em fase gasosa em recipiente fechado, ou a uma reação em fase líquida num reator contínuo com agitação), por forma a garantir homogeneidade. Com estas hipóteses, o modelo pode ser descrito, em linguagem da Cinética Química, pelo seguinte esquema:

$$
S+I \stackrel{k_{1}}{\longrightarrow} 2 I
$$

$$
I \stackrel{k_{2}}{\longrightarrow} R
$$

0 primeiro passo tem em conta o processo de transmissão da infeção, que se dá por encontro de um infetante com um suscetível, sendo $k_{1}$ a constante de velocidade do processo de infeção (que se admite não variar no tempo, como pode suceder através de mutações no agente patogénico ou de intervenção humana sobre as condições de transmissão). Traduz a frequência de contactos e ainda a probabilidade de transmissão ainda hoje importante, foi proposto por Kermack e McKendrick há quase um século [11] e é conhecido 
num contacto, de forma semelhante à da constante de velocidade de uma reação bimolecular elementar, tal como formulada pela Teoria das Colisões, que em solução se escreve $k_{1}=p k_{d}$, em que $k_{d}$ é a constante de velocidade para um processo controlado pela difusão e pé a probabilidade de transmissão (reação) num encontro. Porém, ao contrário de uma reação elementar, em que se verifica o princípio da reversibilidade microscópica, este processo é completamente irreversível. 0 segundo passo representa a imunização ou morte dos infetantes, que se tornam dessa forma elementos da categoria dos removidos, precisamente por serem retirados do processo de contágio, sendo $k_{2}$ a constante de velocidade de remoção, e que é uma probabilidade de transição por unidade de tempo. 0 sistema de equações de velocidade correspondentes ao mecanismo é, assim [11-14]:

$\frac{d S}{d t}=-k_{1} S I$

$\frac{d I}{d t}=k_{1} S I-k_{2} I=k_{2} I\left(\frac{k_{1} S}{k_{2}}-1\right)$

$\frac{d R}{d t}=k_{2} I$

Uma vez que $R$ não figura nas duas primeiras equações, o problema reduz-se, na prática, a um sistema de duas equações diferenciais (autónomas e não lineares). A dinâmica é determinada por quatro parâmetros: (i) Número inicial de suscetíveis, $S_{0}$; (ii) Número inicial de infetantes, $I_{0}$ (que se supõe serem misturados com os suscetíveis no instante inicial). I é normalmente muito menor do que $S_{0}$ (pode mesmo ser um único indivíduo, veja-se o exemplo da Secção 3); (iii) Constante de velocidade $k_{1}$ que reflete a probabilidade de transmissão; (iv) Constante de velocidade $k_{2}$ que reflete a duração média de um infetante (é tanto maior quanto menor esta for).

Supõe-se habitualmente, sem perda de generalidade, que não há inicialmente elementos da categoria dos Removidos. Assim, $R$ é dado por $R$ $=S_{0}+I_{0}-(S+I)$. Considerando a duração típica de uma epidemia, os processos de nascimento e morte (por outras causas) bem como emigrações e imigrações, podem ser desprezados.

Nas equações cinéticas, o número de elementos de cada classe deve, em rigor, ser substituído por uma concentração (por exemplo densidade populacional para a categoria em questão) mas, dado que a área (ou o volume) são fixos, este efeito é incorporado no valor da constante de velocidade $k_{1}$. Apenas quando se pretende comparar sistemas diferentes é necessário ter isto em conta.

Se os infetantes não chegassem a ser misturados com os suscetíveis no instante zero, o seu número decresceria com o tempo segundo uma lei exponencial:

$$
I=I_{0} \exp \left(-k_{2} t\right)
$$

O período infeccioso médio, $\tau$, que é também o tempo médio de recuperação de um infetante (desprezando a possibilidade de morte) é definido por:

$\tau=\frac{1}{k_{2}}$

que é o tempo de decaimento da eq. 16.

A grandeza $E=k_{1}$ SI é muito importante, pois significa o número de novos casos (infetantes) por unidade de tempo (normalmente por dia), como resulta da eq. 14 . Ao seu andamento, $E(t)$, dá-se o nome de curva epidemiológica $[13,14]$. A curva dos infetantes, $I(t)$, tem sempre um atraso em relação a esta, pois o efeito dos novos casos é cumulativo [15].

A grande diferença do mecanismo em análise em relação ao da autocatálise pura (sem inibição) é o facto de o número de infetantes não aumentar necessariamente com o tempo. 0 resultado é claro considerando a eq. 14: para a derivada ser positiva a tempo zero, tem de se verificar que

$\frac{k_{1} S_{0}}{k_{2}}>1$

havendo, portanto, um valor mínimo de suscetíveis, dito limiar ou crítico, $S_{c}[11-13]$ :

$S_{0}>S_{c}=\frac{k_{2}}{k_{1}}$

Se o número inicial de suscetíveis for inferior a este valor, o número de infetantes diminui com o tempo, e a epidemia não acontece (embora possa haver um número apreciável de infetados subsequentes). A explicação é, obviamente, que a velocidade de remoção supera a velocidade de produção de infetantes. No artigo original [9], Kermack e McKendrick especulam que a existência do número crítico $S_{c}$ poderia justificar 
que, em certas regiões de doenças contagiosas de letalidade elevada e endémicas, as populações não ultrapassassem um determinado valor.

Outro parâmetro importante é o número básico de reprodução da infeção, $R_{0}$ [12-14], definido como o número médio de suscetíveis infetados por um infetante, antes de este se transformar em removido: É o equivalente ao número médio de ciclos por catalisador (turnover number), aqui também um fator de multiplicação por ser autocatálise. 0 valor refere-se normalmente ao instante inicial, daí o índice.

Esta grandeza adimensional define-se a partir da eq. (18),

$R_{0}=\frac{k_{1} S_{0}}{k_{2}}=\frac{S_{0}}{S_{c}}$

Segue-se desta relação que a epidemia se pode instalar se $R_{0}>1$. Para instantes posteriores, quando $S$ já é inferior a $S_{0}$, ou quando $k_{1}$ varia com o tempo, define-se o parâmetro $R_{\mathrm{t}}$,

$R_{t}=\frac{k_{1} S}{k_{2}}$

Para demonstrar o significado de $R_{0}$ note-se que a velocidade de infeção por infetante é:

$$
\frac{E}{I}=k_{1} S
$$

A tempo zero, esta tem o valor

$$
\frac{E(0)}{I_{0}}=k_{1} S_{0}
$$

Finalmente, usando o período infeccioso médio $\tau$, o número básico de reprodução fica

$$
R_{0}=k_{1} S_{0} \tau
$$

Note-se que este valor é uma média: os infetados por infetante inicial obedecem, em primeira aproximação, a uma distribuição geométrica de média $R_{0}$. Assim, para $R_{0}=2,5$, por exemplo, a probabilidade de um infetado inicial não infetar ninguém antes de passar a removido é de 0,29 . Dos $71 \%$ de infetantes iniciais que de facto infetam, $29 \%$ infetam uma pessoa, $20 \%$ infetam duas, $15 \%$ infetam três, $10 \%$ infetam quatro, $7 \%$ infetam cinco, etc. Conhecem-se, para algumas doenças muito contagiosas, valores de $R_{0}$ que atingem 15 e mais, embora sejam mais comuns valores entre 1,5 e 5 [16]. 0 valor de $R_{0}$ reflete não só os aspetos intrínsecos da doença, mas também as condições específicas da população (frequência de contactos, probabilidade de transmissão). Para a COVID-19 em Wuhan, por exemplo, o valor inicial de $R_{0}$ (janeiro de 2020) foi estimado em 2,35 (ou 2,5 [18]), descendo em duas semanas para 1,05 após a imposição de restrições à circulação [17]. No início da epidemia, quando $S_{0}$ é aproximadamente constante, o número de infetantes cresce exponencialmente,

$$
I=I_{0} \exp \left[\left(k_{1} S_{0}-k_{2}\right) t\right]
$$

Compare-se com a eq. 5, e o tempo de duplicação dos infetantes, $T_{2}$, é dado por

$T_{2}=\frac{\ln 2}{k_{1} S_{0}-k_{2}}=\frac{\tau \ln 2}{R_{0}-1}$

Compare-se com a eq. 6. Esta equação permite estimar $R_{0}$ numa fase inicial da epidemia, caso $\tau$ já seja conhecido a partir de pacientes já recuperados (ou falecidos) [16]:

$R_{0}=1+\ln 2\left(\frac{\tau}{T_{2}}\right)$

Na Tabela 1, apresentam-se valores de $R_{0}$ calculados com parâmetros na gama de aplicabilidade à pandemia da COVID-19 [18]:

Tabela 1 - Valores de $R_{0}$ calculados com a eq. 27

\begin{tabular}{|c|c|c|c|c|}
\hline \multicolumn{2}{|c|}{} & \multicolumn{3}{|c|}{$\tau / \mathbf{d}$} \\
\cline { 2 - 5 } \multicolumn{2}{|c|}{} & $\mathbf{7}$ & $\mathbf{1 4}$ & $\mathbf{2 1}$ \\
\hline \multirow{3}{*}{$\mathbf{T}_{2} / \mathbf{d}$} & $\mathbf{2}$ & 3,4 & 5,9 & 8,3 \\
\cline { 2 - 5 } & $\mathbf{3}$ & 2,6 & 4,2 & 5,9 \\
\cline { 2 - 5 } & $\mathbf{4}$ & $\mathbf{2 , 2}$ & 3,4 & 4,6 \\
\cline { 2 - 5 } & $\mathbf{5}$ & 2,0 & 2,9 & 3,9 \\
\cline { 2 - 5 } & $\mathbf{6}$ & 1,8 & 2,6 & 3,4 \\
\hline
\end{tabular}

A eq. 27 tem também interesse para avaliar a rapidez de progressão inicial de uma epidemia, conhecidos o $R_{0}$ e o $\tau$. Por exemplo, se $R_{0}=2,5$ e $\tau=7 \mathrm{~d}$, vem $T_{2}$ $=3,2 \mathrm{~d}$. Isto explica a necessidade de as medidas de contenção serem tomadas com rapidez. 
Para combater uma epidemia, e extingui-la, se possível, é necessário baixar o valor de $R_{0}$ (aumentar o valor de $S_{c}$ ) através de: (i) Decréscimo do valor de $k_{1} S_{0}$, por redução da frequência de contacto (isolamento de suscetíveis e de infetantes, quarentena) e da eficiência de infeção no contacto (distanciamento social, medidas de proteção pessoal, aumento da higiene pessoal e do meio, tanto para suscetíveis como para infetantes); (ii) Aumentar o valor de $k_{2}$, por tratamento médico dos infetantes (sendo a outra razão a preservação das suas vidas).

A vacinação em massa também tem uma importância grande, pois traduz-se por uma transferência direta da categoria dos Suscetíveis para a categoria dos Removidos, sem passar pela categoria dos Infetantes, reduzindo simultaneamente o valor de S. É, assim, possível descer abaixo do valor crítico, tornando a epidemia inviável por se ter atingido a imunidade de grupo, e isto sem que haja, sequer, infetados. Por exemplo, no caso de uma vacinação de frequência constante, e com início a tempo zero, o sistema de equações de velocidade fica:

$\frac{d S}{d t}=-k_{1} S I-k_{3}$

$\frac{d I}{d t}=I\left(k_{1} S-k_{2}\right)$

$\frac{d R}{d t}=k_{2} I+k_{3}$

sendo $k_{3}$ a velocidade de vacinação, que aqui corresponde a um processo de ordem zero.

Deve notar-se que o modelo SIR não tem memória: a sua dinâmica não é afetada pelos estados anteriores, e uma alteração nos parâmetros (e.g. $k_{1}$ ) ou condições (e.g. vacinação) num dado momento, produz efeitos a partir desse momento. Define-se, pois, uma nova origem dos tempos (ver o exemplo na Secção 3).

A entrada (ou saída) de infetantes no sistema (por exemplo viajantes) pode ser traduzida por uma função de entrada/saída $A(t)$,

$\frac{d I}{d t}=A(t)+I\left(k_{1} S-k_{2}\right)$

Consideremos o caso mais simples (sem entrada ou saída de infetantes nem vacinação), tal como feito por Kermack e McKendrick, eqs. 13-15. Devido aos termos não lineares, não é possível obter uma solução analítica explícita para o sistema de equações diferenciais, e a evolução temporal tem de ser obtida por integração numérica ou recorrendo a aproximações. São, contudo, possíveis alguns resultados analíticos, como se discute de seguida.

A eliminação do tempo das eqs. 13 e 14 dá [11-14]

$\frac{d I}{d S}=\frac{S_{c}}{S}-1$

cuja integração conduz a uma relação entre I e S:

$I=I_{0}+S_{0}-S+S_{c} \ln \left(\frac{S}{S_{0}}\right)$

Este é um dos resultados básicos do modelo. 0 valor máximo de $/$ dá-se para $S=S$, como se segue da eq. 32, ou, em termos de parâmetros reduzidos (v. Figura 3),

$\frac{S_{c}}{S_{0}}=S_{c}=\frac{1}{R_{0}}$

A eq. 33 leva a [11-14]

$I_{\text {max }}=I_{0}+S_{0}-S_{c}+S_{c} \ln \left(\frac{S_{c}}{S_{0}}\right)$

que pode ser reescrita como

$i_{\text {max }}=1+i_{0}-\frac{1}{R_{0}}\left(1+\ln R_{0}\right)$

sendo $i_{\max }=I_{\max } / S_{0}$ e $i_{0}=I_{0} / S_{0}$. Neste ponto de viragem da epidemia, em que se atinge a imunidade de grupo, o número de removidos é

$R_{\max }=I_{0}+S_{0}-I_{\text {max }}-S_{c}=-S_{c} \ln \left(\frac{S_{c}}{S_{0}}\right)$

ou

$\frac{R_{\max }}{S_{0}}=\frac{\ln R_{0}}{R_{0}}$ 
A fração relativa de cada uma das classes no pico da epidemia, eqs. 31, 33 e 35, é apresentada na Figura 2 para $i_{0}<1$. Para $R_{0}$ ligeiramente acima de 1 , a fração de infetantes é muito pequena, mas aumenta de forma pronunciada com $R_{0}$, excedendo mesmo as das outras duas categorias para $R_{0}>3,5$.

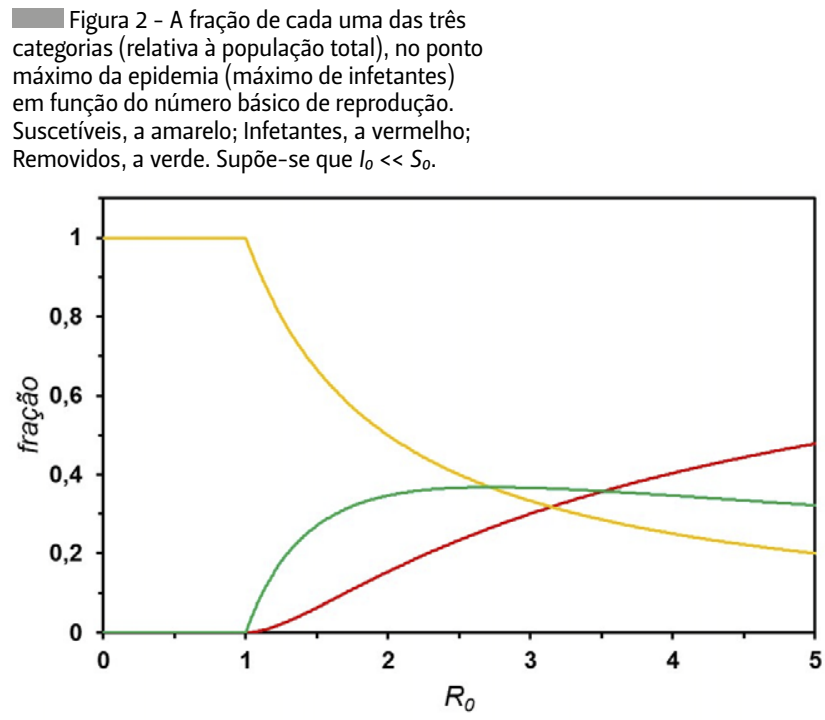

Observa-se ainda que, para valores elevados de $R_{0}$, a fração de removidos diminui com $R_{0}$. Desta forma, $R$ é desprezável para $R_{0}$ alto, e a curva logística, eq. 4, aplica-se a Se a I.

É útil ter uma expressão explícita para S em função de I, isto é, resolver a eq. 33 em ordem a S. Isto só é possível fazendo uso da função de Lambert, $W(x)$ [19]:

$\frac{S}{S_{0}}=-\frac{1}{R_{0}} W_{-1}\left(-R_{0} e^{-\left(1+i_{0}-i\right) R_{0}}\right) \quad$ se $S \geq S_{c}$

$\frac{S}{S_{0}}=-\frac{1}{R_{0}} W_{0}\left(-R_{0} e^{-\left(1+i_{0}-i\right) R_{0}}\right) \quad$ se $\quad S \leq S_{c}$

sendo $W_{0}(x)$ a função calculada no seu ramo principal e $W_{-1}(x)$ a função calculada no ramo secundário. Usando as eqs. 39 e 40, pode calcular-se diretamente o valor de $\mathrm{S}$ em qualquer ponto da epidemia. Por exemplo, quando os infetantes atingem o seu valor máximo, a eq. 36 é válida e, substituída na eq. 39 ou na eq. 40, dá $S_{\max } / S_{0}=1 / R_{0}$, como esperado. 0 valor final de $S$ (no fim da epidemia), $S_{\infty}$, também se obtém da eq. 40 , desta vez fazendo $i=0$ :
$\frac{S_{\infty}}{S_{0}}=-\frac{1}{R_{0}} W_{0}\left(-R_{0} e^{-\left(1+i_{0}\right) R_{0}}\right)$

Admitindo que $I_{0} \ll S_{0}$, como é normalmente o caso, é possível ajustar uma função simples e precisa (para $R_{0}>1$ ) [19], com o comportamento assimptótico correto:

$\frac{S_{\infty}}{S_{0}}=\exp \left(-R_{0}\right)+2,462 \exp \left(-1,851 R_{0}\right)+8,798 \exp \left(-3,580 R_{0}\right)$

A dependência de $S_{\infty} / S_{0}$ com $R_{0}$ está representada na Figura 3. Como já referido, para $R_{0}<1$ não há epidemia, e o número de suscetíveis não diminui significativamente (admitindo $I_{0} \ll S_{0}$ ). A fração de população que não contrai a doença é de $40 \%$ para $R_{0}=1,5,20 \%$ para $R_{0}=2$, e de $6 \%$ para $R_{0}=3$. Para $R_{0}>5$, praticamente toda a população é infetada. Representa-se na mesma figura a fração de suscetíveis necessária para se atingir a imunidade de grupo, $S_{c} / S_{0}$, eq. 34. Atingida esta fração, embora a doença se continue a propagar, o número de infetantes começa a diminuir. Para $R_{0}=2,5$, por exemplo, a fração crítica de suscetíveis é de 0,4, ou seja, $60 \%$ da população já terá sido infetada no instante em que se atinge a imunidade de grupo. Isto não quer dizer que a epidemia acabe nesse momento, apenas se passa a propagar cada vez mais devagar. Com efeito, a eq. 42 (v. também Fig. 3) indica que, no fim da epidemia, apenas $11 \%$ da população terá escapado à doença, ou seja, após a passagem do ponto crítico ainda há mais 29\% da população que fica infetada. à doença, $S / S_{0}$, em função do número básico de reprodução, $R_{0}$ (eqs. 41 e 42 , linha contínua). Fração de suscetíveis correspondente à imunidade de grupo (eq. 34, linha tracejada). Admite-se que $I_{0} \ll S_{0}$.

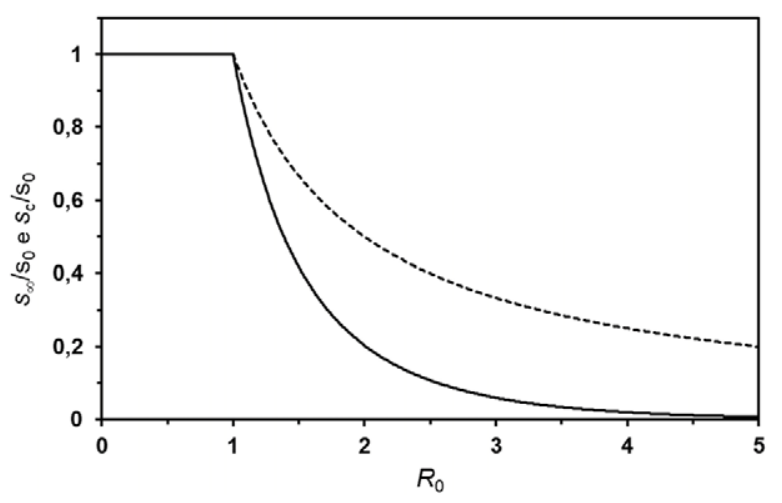




\section{Exemplo de aplicação do modelo SIR}

Como primeiro exemplo de aplicação, considere-se um caso simples e elucidativo [20], citado num livro de texto [12]: um surto de gripe A (vírus H1N1) num colégio interno inglês, em janeiro-fevereiro de 1978 [20]. A origem desta pandemia de ǵripe em 1977/8, que atingiu principalmente jovens com menos de 20 anos por o vírus ser recorrente (1957 e anos anteriores), foi atribuída a ensaios descontrolados de vacinação em militares chineses [21]. No colégio existiam 763 rapazes com idades entre 10 e 18 anos, sendo a grande maioria internos. Após as Férias de Natal, um dos alunos, regressado de Hong-Kong, apresentou sintomas de gripe. Ao fim de uma semana, surgiram mais casos. 0 número de alunos acamados (durante 3 a 7 dias, depois convalescentes) é dado na Tabela 2, obtido a partir do gráfico publicado [20]:

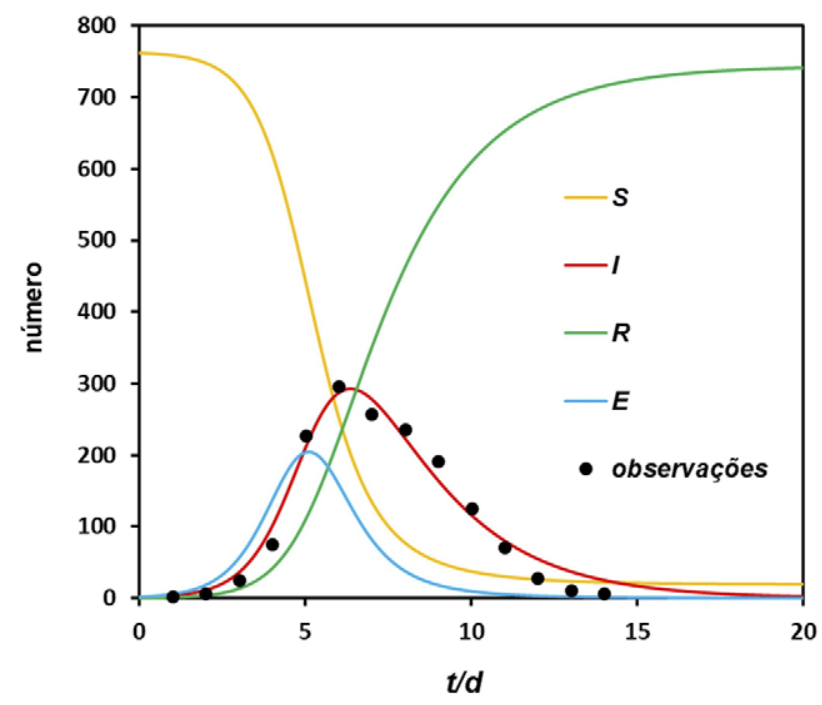

Figura 4 - Exemplo do surto no colégio interno [20]: observações e curvas do modelo.

Tabela 2 - Número de infetantes em cada dia

\begin{tabular}{|l|c|c|c|c|c|c|c|c|c|c|c|c|c|c|}
\hline Dia & 1 & 2 & 3 & 4 & 5 & 6 & 7 & 8 & 9 & 10 & 11 & 12 & 13 & 14 \\
\hline Infetantes & 3 & 6 & 25 & 75 & 227 & 296 & 258 & 236 & 192 & 126 & 71 & 28 & 11 & 7 \\
\hline
\end{tabular}

Estes alunos são considerados infetantes, presumivelmente por não estarem isolados. Dos cerca de 130 adultos do colégio, apenas um teve sintomas.

A partir de um ajuste pelo método dos mínimos quadrados não linear, recorrendo a integração numérica com $I_{0}=1$ e $S_{0}=762$, obtiveram-se as seguintes constantes de velocidade: $k_{1}=2,2 \times 10^{-3}$ pessoa $^{-1} \mathrm{~d}^{-1}$ e $k_{2}=0,44 d^{-1}$. Estes valores estão em completa concordância com os apresentados em [12]. Com os parâmetros determinados, obtiveram-se as curvas dadas na Figura 4, e os parâmetros da Tabela 3 (com incertezas associadas de alguns \%). Observa-se uma boa concordância geral, embora não perfeita, mas não se pode esperar mais do modelo. 0 número de novos casos por dia (E) terá atingido cerca de 205 no $5^{\circ}$ dia. 0 máximo desta curva antecede sempre o máximo da curva dos infetantes (I), como se referiu [15]. 0 número total de infetantes chegou a ser de 293 no $6^{\circ}$ dia (40\% do total de alunos). Ao fim de duas semanas, o surto estava praticamente extinto, tendo atingido cerca de $98 \%$ dos alunos. 0 parâmetro $R_{0}(3,8)$ indica uma situação de rápida e extensa propagação, como se verificou.
Uma outra forma de apresentar os resultados é a representação no espaço das fases, Figura 5, em que o estado do sistema num dado instante corresponde ao ponto $(S, I)$. O sistema evolui da direita para a esquerda, segundo a eq. 33. Os pontos 1 a 4 assinalam as várias fases do surto.
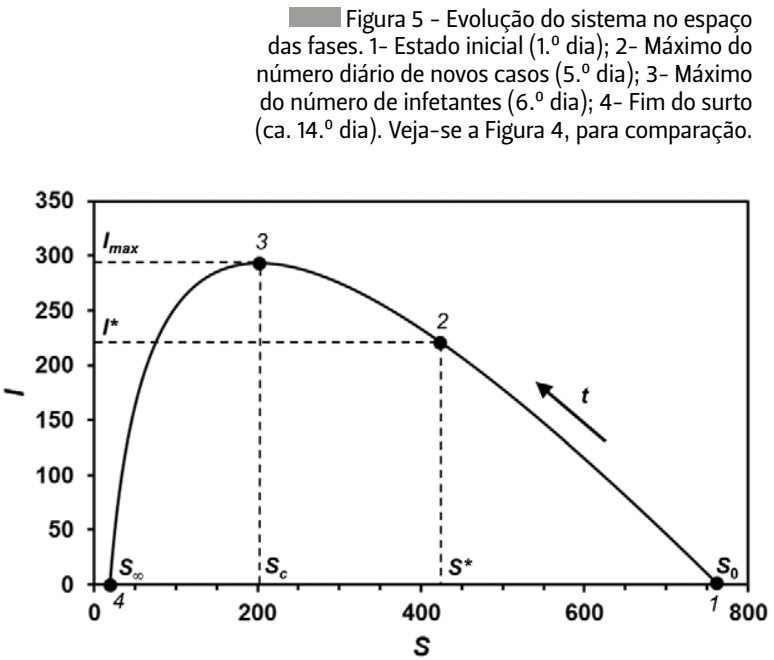

Tabela 3 - Parâmetros e grandezas calculados

\begin{tabular}{|l|c|c|c|c|c|c|c|c|c|c|}
\hline Grandeza ou parâmetro & $T_{2} / \mathrm{d}$ & $\tau / \mathrm{d}$ & $S_{c}$ & $R_{0}$ & $t^{*} / \mathrm{d}$ & $E^{*}$ & $S^{*}$ & $t_{\max } / \mathrm{d}$ & $I_{\max }$ & $S_{\infty}$ \\
\hline Valor & 0,57 & 2,3 & 202 & 3,8 & 5,1 & 205 & 420 & 6,4 & 293 & 19 \\
\hline
\end{tabular}


Tem interesse ver o que se poderia ter passado, se tivesse havido um maior número inicial de infetantes. Na Figura 6 considera-se $I_{0}=20$. A existência inicial de 20 casos faz com que o tempo para se atingir o máximo de I diminua apreciavelmente, passando para 3,9 dias (diminuição do tempo de indução), mas apenas subindo $0 I_{\text {max }}$ de forma moderada, para 313 (eq. 35). A situação é quase equivalente a ir procurar no caso original o instante em que $I=20$, e fazer dele a nova origem dos tempos. Isto sucede assim pois $S$ quase não se afasta de $S_{0}$ nesse intervalo, o que já não acontece para valores de $I_{0}$ muito mais elevados. Este exemplo calculado mostra que se o número inicial de infetantes for reduzido, o seu efeito é muito semelhante, independentemente de quantos forem. Só o tempo de indução é afetado de forma importante.

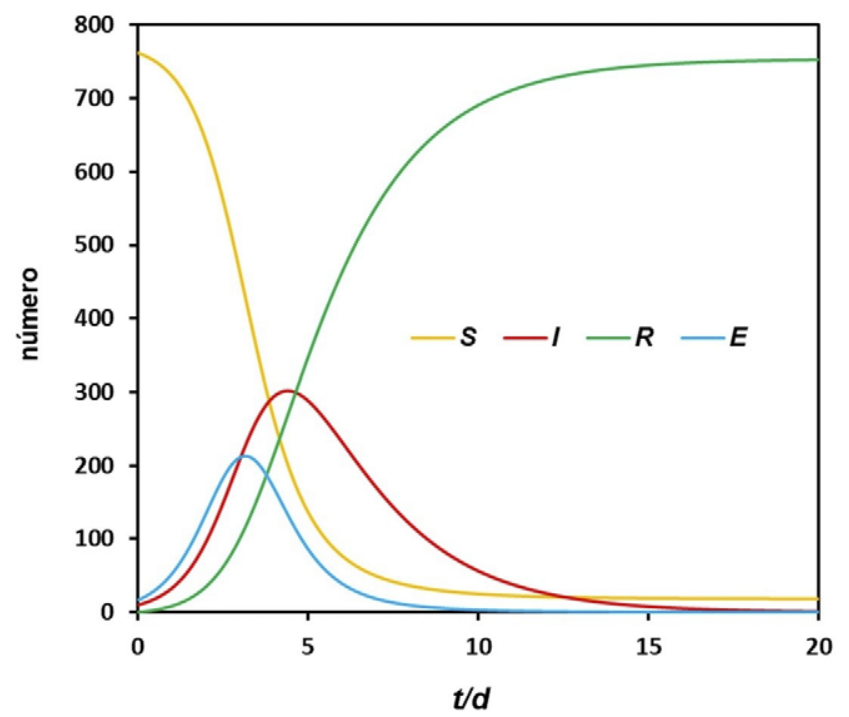

Figura 6 - Parâmetros idênticos aos da Fig. 4, com a exceção de $I_{0}=20$.

Também se pode estudar o efeito de uma redução intencional de $k_{1}$ durante o surto (o chamado «achatamento da curva»). Suponhamos que, no 4. ${ }^{\circ}$ dia $(S=621)$, devido a medidas de contenção e isolamento, $k_{1}$ desce para metade do valor inicial, Figura 7. 0 novo $R_{0}$, calculado para essa nova origem dos tempos (usando $S=621$ ), seria de 1,5 (em vez de 3,1 , sem alteração de $k_{1}$ ), portanto ainda há subida $\left(R_{0}>1\right)$, mas menos pronunciada. Como se vê, o valor de $I_{\max }(143)$ desce para cerca de metade, e o número de suscetíveis que não contraem a doença (153) aumenta muito. Contudo, pode não ser possível manter as medidas de contenção indefinidamente (neste exemplo a população é pequena, e os tempos também; não é assim noutros casos).

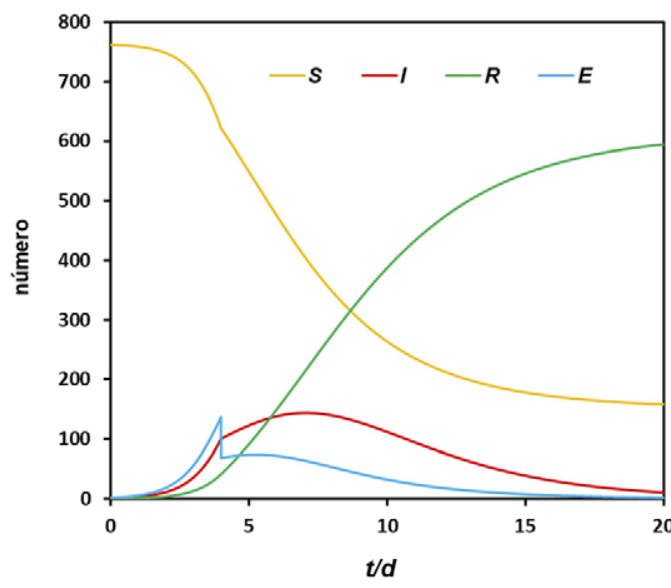

Figura 7 - Idêntico à Fig. 4, mas com redução brusca de $k_{1}$ para metade a partir do $4 .{ }^{\circ}$ dia.

Que sucederia se, no $10 .^{\circ} \mathrm{dia}(S=266)$, as medidas de contenção fossem abolidas e o $k_{1}$ voltasse ao valor inicial? É o que se mostra na Figura 8. Não haveria novo surto significativo, pois o $R_{0}$ seria ligeiramente superior a $1\left(S_{c}=202\right)$, mas o número de suscetíveis que não contraem a doença (54) diminuiria consideravelmente, em relação ao caso anterior.

Na Figura 9 considera-se uma situação semelhante, mas em que as medidas de contenção foram mais drásticas, e a constante $k_{1}$ caiu para um décimo do valor inicial. Nesse caso o $R_{0}$ passa para 0,31, situação (muito) subcrítica. 0 número de infetantes decai exponencialmente (com um tempo de vida $\tau$ ), e o de suscetíveis mantém-se praticamente constante. Assim, a situação fica «congelada», mantendo-se o potencial epidémico, uma vez que $S$ permanece muito acima do valor crítico, $S_{c}$. Em consequência, quando se levantam as medidas de contenção (10. dia), e o k, volta ao valor inicial, dá-se um novo surto (a "segunda vaga"), que só esmorece quando S desce, finalmente, abaixo do valor crítico.

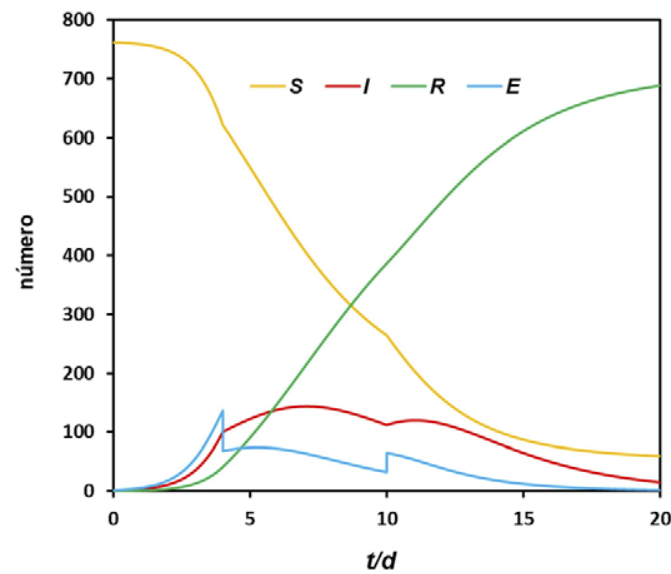

Figura 8 - Idêntico à Fig. 7, mas com reposição do $k_{1}$ inicial no $10 .^{\circ}$ dia. 


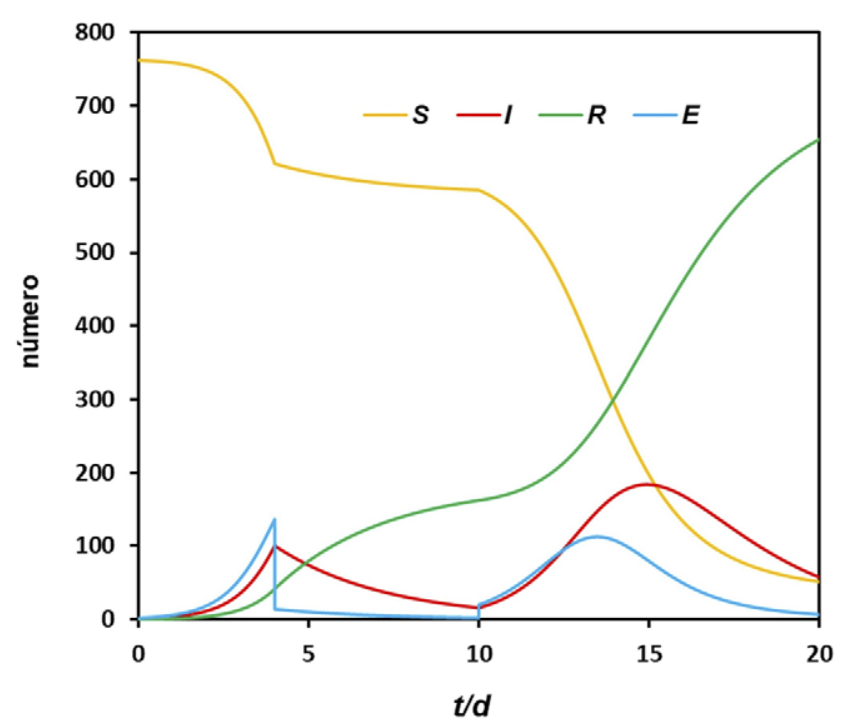

Figura 9 - Semelhante à Fig. 8 , mas com redução de $k_{1}$ para $1 / 10$ entre o $4 . .^{\circ}$ dia e o $9 .{ }^{\circ}$ dia, e reposição do valor inicial no $10 .^{\circ}$ dia.

Na ausência de vacina ou terapia eficaz, uma boa forma de converter os suscetíveis em removidos é, pois, manter o $R_{t}$ (eq. 21) constantemente igual a 1, o que permite fixar o número máximo de infetantes. Isto implica que, depois de um corte significativo inicial, se aumente progressivamente $k_{1}$, aproximando-o do valor inicial (vida sem restrições), à medida que o $S$ vai diminuindo. É o que se exemplifica nas Figuras 10 e 11. Fixando, por exemplo, um limite superior para os infetantes de 100, assim que este número é atingido, a constante de velocidade $k_{1}$ é reduzida para um valor que impede mais crescimento, ou seja, para que o $R_{t}$ se fixe em 1, o que é conseguido com

$$
k_{1}(t)=\frac{k_{2}}{S}
$$

Deste modo, $k_{1}$ vai subindo progressivamente, até que, quando $S=S_{c}$, iguala o valor original (valor "natural"), Figura 10, acabando nesse momento as medidas restritivas.

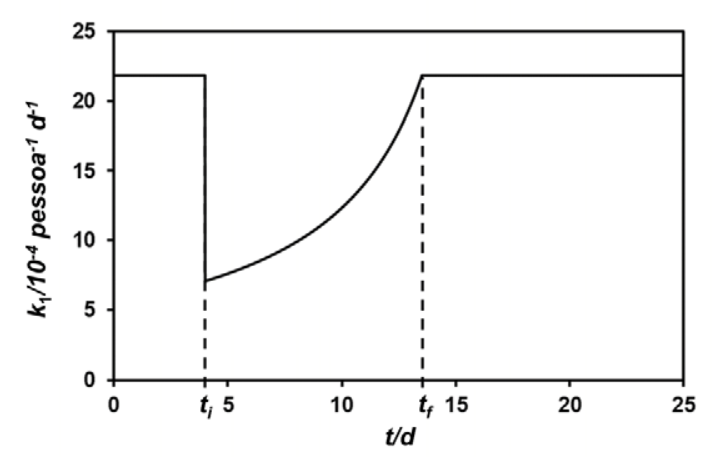

Figura 10 - Evolução de $k_{1}$, reduzido pelas medidas restritivas entre $t_{i}$ ( 4 dias) e $t_{f}$, (14 dias).
Isto evita qualquer subida posterior do número de infetantes, e tem-se a evolução que se apresenta na Figura 11, em que o período com restrições é o menor possível (neste caso cerca de 10 dias). Note-se que tanto $S$ como $R$ variam linearmente no período em que lé constante (como resulta da eq. 13 e da constância do número total de pessoas), e, portanto, $k_{1}$ varia nesse período de forma hiperbólica (v. eq. 43 e Fig. 10).

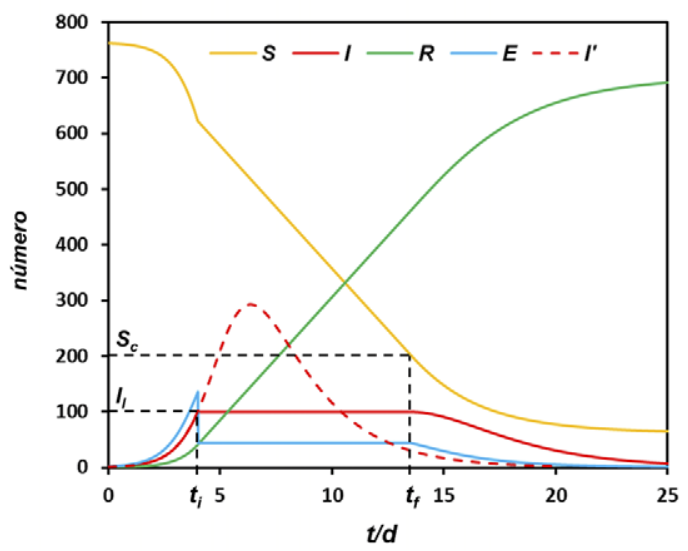

Figura 11 - Evolução do sistema com controlo da constante de velocidade $k_{1}$ de tal forma que nunca ultrapassa o limite $I_{i}$ (neste caso 100). Também se representa, para comparação, a evolução original de I( $I$, linha vermelha a tracejado), sem controlo (compare-se com a Fig. 4).

0 número de suscetíveis não infetados, $S_{\infty}$, pode ser calculado usando a eq. 41, mas em que $S_{0}$ é substituído por $S_{C^{\prime}}$ e $i_{0}$ é substituído por $I_{i} / S_{C}$, o que dá 61 pessoas.

\section{O modelo SIR e a COVID-19}

0 modelo SIR, puramente determinístico, baseia-se num certo número de aproximações drásticas, designadamente que cada categoria (em especial S e I) tem, desde o início, uma distribuição espacial homogénea. Contudo, exceto para situações como a do colégio apresentada acima, uma descrição mais realista será a de um conjunto de compartimentos comunicantes, cada um com a sua densidade e números iniciais, situação mais próxima de um sólido poroso ou de uma rede de reatores do que de um só reator macroscópico. De notar que a divisão por categorias não implica uma «reatividade» única. Mesmo no caso molecular, a constante $k_{1}$ resulta de uma média sobre energias (distribuição de Boltzmann, contudo existe ergodicidade, ou seja, cada molécula vai passando pelos vários estados energéticos).

$\mathrm{Na}$ forma mais simples, admite-se ainda que a constante $k_{1}$ não varia com o tempo (mas no exemplo acima considerou-se o efeito dessa variação).

Como uma primeira aproximação, que representa um compromisso variável entre rigor e simplicidade, 
o modelo SIR pode ser aplicado a regiões, países ou, até, no limite dos limites, ao mundo!

Um aspeto importante que distingue a Cinética Química da Epidemiologia é a qualidade dos dados experimentais. Numa área as experiências são controladas e podem repetir-se, na outra há muitas incertezas envolvidas na obtenção dos dados, e a repetição é impossível (pelo menos com seres humanos). O número registado de infetados, por exemplo, depende, no caso da COVID-19, do número de testes realizados, e é um minorante do número real (que pode ser estimado, mas não obtido diretamente, na maioria dos casos). Em certos países os números são baixos não só por falta de testes (deliberadamente ou por escassez de meios) mas também por os resultados, suspeita-se, serem manipulados. Mesmo o número de mortes pode ser inferior ao real, ao serem atribuídas a outras causas. O contrário também é possível, como sucedeu em Espanha, em que havia quase duas mil mortes a mais, depois removidas em bloco das estatísticas. Também as datas em que são feitos os registos podem diferir das datas verdadeiras (diferenças de alguns dias). Em todo o caso, o número de mortes (quando em número significativo) parece ser um conjunto de dados relativamente robusto, pelo menos para alguns países.

A eq. 14 permite calcular $R_{\mathrm{t}}$,

$R_{t}=1+\frac{1}{k_{2} I} \frac{d I}{d t}$

Infelizmente, o número de infetantes e a sua variação diária não são fáceis de estimar. Por outro lado, a eq. 15 relaciona o número de novos removidos por dia, $\dot{R}=\frac{d R}{d t}$, com o número de infetantes, I. Admitindo que o número de mortes por dia, $\dot{M}=\frac{d M}{d t}$, é uma fração fixa do número diário de removidos, $\dot{R}$, (hipótese razoável no âmbito do modelo SIR), segue-se que $\dot{M}$ e I são proporcionais, donde

$$
R_{t}=1+\frac{1}{k_{2} \dot{M}} \frac{d \dot{M}}{d t}=1+\tau \frac{d \ln \dot{M}}{d t}
$$

Esta equação permite estimar o valor de $R_{\mathrm{t}}$ em função do tempo, desde que se admita um valor para $k_{2}$. Como exemplos, nas Figuras 12-14 e 15-18 apresentam-se os valores de $\dot{M}$ [22] e de $R_{\mathrm{t}}$ para Portugal, Itália e EUA.
Os valores de $R_{t}$ calculados para Portugal podem ser comparados com os dados pelo INSA, estimados com um modelo mais elaborado [25].
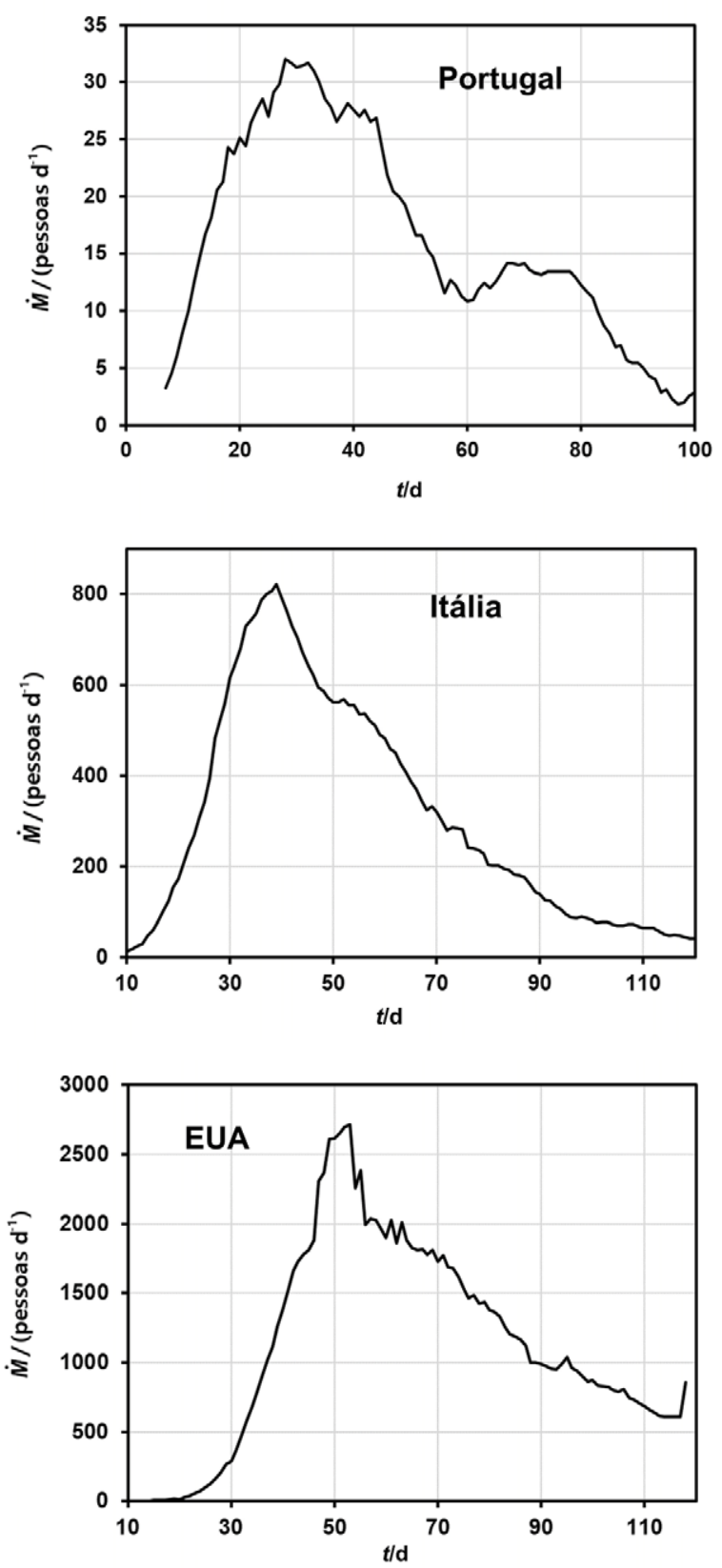

Figuras 12-14 - Número diário de mortes por COVID-19 [22], $\dot{M}=\mathrm{dM} / \mathrm{dt}$, para Portugal, Itália e EUA, sendo o último ponto correspondente ao dia 25 de junho de 2020.

Apresentam-se médias móveis de 7 dias.

Verifica-se, nos três casos, que o $R_{t}$ inicial (antes de medidas de contenção) está próximo do valor referido em vários estudos. Note-se que a variação de $k_{2}$ leva a uma compressão ou dilatação vertical da curva $R_{t}$ (sempre em relação a 1), mas não altera o 
andamento geral, que é, provavelmente, o resultado mais interessante da representação, pois traduz a variação da mortalidade, em termos relativos $(\ddot{M} / \dot{M}$. De notar que, em todos estes casos, a fração de população infetada ou que já esteve infetada (1-S/So) estará, muito aproximadamente, entre 2\% (Portugal)e $6 \%$ (Itália) (sendo a letalidade da doença da ordem de 1\% [23], o número é de cerca de 100 vezes o número total de óbitos - contudo há diferenças, dependendo, por exemplo, da estrutura etária de cada país e do nível dos cuidados de saúde). As variações observadas de $R_{t}$ ao longo do tempo resultam principalmente de mudanças em $k_{1}$ (uma vez que $S$ pouco variou), em consequência de medidas quer de contenção (diminuição), quer de desconfinamento (aumento). 0 regresso ao estilo de vida anterior à pandemia levaria também a um regresso ao $R_{0}$ inicial (cerca de 2,5 ).
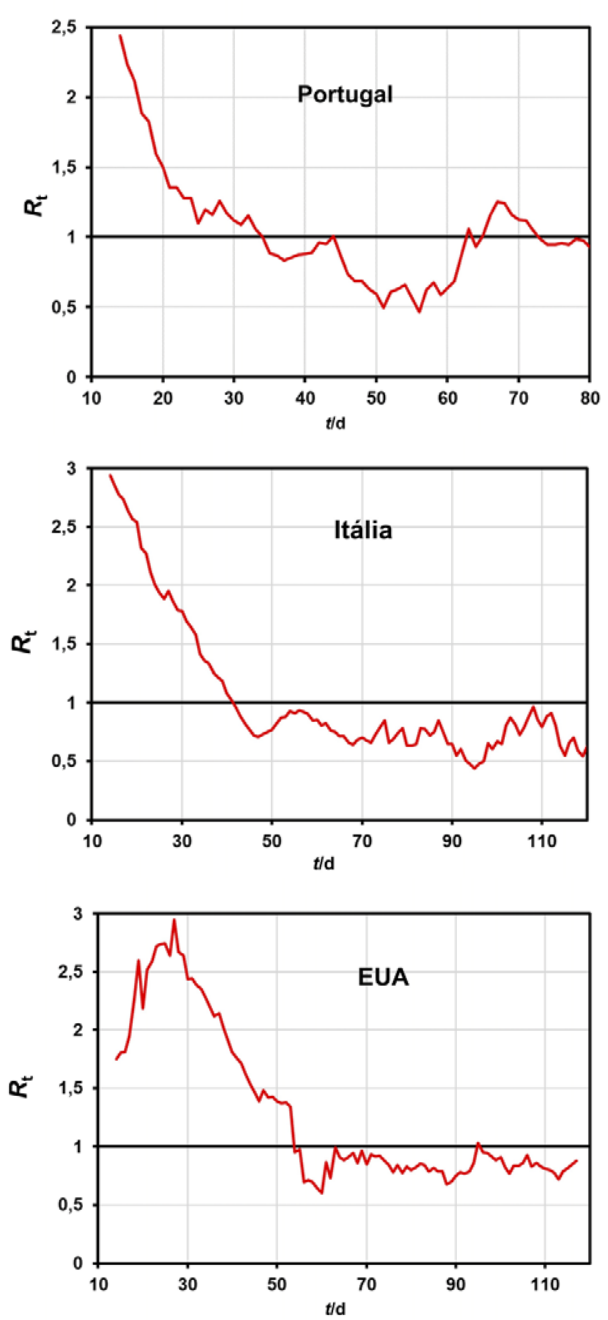

Figuras 15-17 - Parâmetro $R_{t}$ calculado com a eq. 45 para Portugal, Itália e EUA, sendo o último ponto correspondente ao dia 5 de junho (Portugal, último dia com $\dot{M} \geq 10$ pessoas/d) e 25 de junho de 2020 (Itália e EUA). Usa-se um tempo médio de recuperação $1 / k_{2}=7$ dias e médias móveis de 7 dias.
Como mencionado acima (v. Fig. 3 e respetiva discussão), a imunidade de grupo é, segundo o modelo SIR, atingida quando $S / S_{0}=1 / R_{0}$. No caso da COVID-19, usando $R_{0}=2,5$, obtém-se 0,4 , ou seja, $60 \%$ da população precisaria de já ter tido a doença. Certas estimativas usam um $R_{0}$ mais elevado $(3,0)$, e chegam a $67 \%$ [26]. Qualquer destes valores é provavelmente um majorante da percentagem real, cujo valor exato, desconhecido, depende de vários fatores e variará de caso para caso. Num estudo teórico recente [27], em que se considera a heterogeneidade da população, aponta-se para um valor indicativo de $43 \%$.

\section{Conclusões}

Existem muitos pontos de contacto entre a Cinética Química e a Dinâmica Epidemiológica. No que diz respeito à abordagem determinística, ficou aqui estabelecido que a autocatálise com inibição é matematicamente idêntica ao modelo epidemiológico SIR de Kermack-McKendrick. A autocatálise sem inibição, que possui solução analítica (a curva logística), e também tem aplicações em dinâmica populacional, é uma boa forma pedagógica de introduzir o problema. Após estabelecer a semelhança entre as duas áreas científicas, discutiu-se o modelo SIR de Kermack-McKendrick, que foi, por fim, ilustrado por aplicação a um caso concreto e simples de epidemia viral, citado em livros de texto, e que pode ser usado em sala de aula, como ponto de partida para se estudar variantes por efeito de alteração de parâmetros. Usaram-se também alguns resultados que foram aplicados a casos concretos da pandemia da COVID-19 em três países, salientando-se as aproximações feitas.

Do ponto de vista da analogia química estabelecida, há duas situações distintas. Na primeira, caso passivo, o sistema evolui de acordo com os parâmetros iniciais, até que a reação se completa (por esgotamento de infetantes) e não há qualquer intervenção. Trata-se de uma reação a temperatura constante, e o químico (epidemiologista) é apenas um observador interessado (ou o estudioso de um evento já ocorrido). Na segunda situação, caso ativo, há uma intervenção no decurso da reação. Com efeito, o controlo da constante de velocidade $k_{1}$ (medidas de contenção e desconfinamento) pode ser entendido como uma programação de temperatura, desde que se admita que o passo elementar $I \rightarrow R$ é essencialmente não ativado, isto é, independente da temperatura [24]. O valor "natural" de $k_{1}$ corresponderá, digamos, à temperatura ambiente, e o controlo de $k_{1}$ consiste em arrefecer e posteriormente reaquecer a mistura reacional, em grau e duração variáveis, por exemplo para manter o número de infetantes constante (estado estacionário). De facto, as medidas de contenção podem ser descritas como um "arrefecimento" - necessário - da população (e, colateralmente, da economia) pelo governo [28]. 
Considerando a atual situação, nacional e mundial, o paralelo aqui apresentado pode dar origem a discussões e a vários tipos de projetos pedagógicos e, ainda, contribuir para um melhor entendimento da situação e das incertezas associadas.

\section{Agradecimentos}

Trabalho desenvolvido no âmbito do projeto UIDB/04565/2020, Fundação para a Ciência e a Tecnologia (FCT), Portugal.

\section{Referências}

[1] P. Gray, S. K. Scott, Chemical Oscillations and Instabilities. Non-linear Chemical Kinetics, Clarendon Press, Oxford, 1990.

[2] R. Plasson, A. Brandenburg, L. Jullien, H. Bersini, J. Phys. Chem. A 115 (2011) 8073-8085.

[3] M. Garcia-Moreno, B. H. Havsteen, R. Vardn, H. Rix-Matzen, Biochim Biophys. Acta 1080 (1991) 143-147.

[4] Deduzida e assim designada por Verhulst $(1838,1845)$ no contexto de um modelo de crescimento populacional, veja-se J. S. Cramer, Tinbergen Institute Working Paper No. 2002-119/4 (2002). doi.org/10.2139/ssrn.360300

[5] S. Kingsland, Quart. Rev. Biol. 57 (1982) 29-52.

[6] T. B. Robertson, Arch. Entwickmech. Organ. 25 (1908) 581-614.

[7] R. Pearl, L. J. Reed, Proc. Natl. Acad. Sci. 6 (1920) 275-288.

[8] Em Demografia, dá-se ao crescimento exponencial o nome de modelo maltusiano. A abordagem de Verhulst foi precisamente a de corrigir o resultado de Malthus, por inclusão na equação de evolução de um termo quadrático negativo (matematicamente equivalente à inclusão de $S$ na eq. 3), veja-se [4].

[9] A. G. McKendrick, M. K. Pai, Proc. R. Soc. Edinb. 31 (1911) 649-655.

[10] A. L. Koch, The Monod Model and Its Alternatives, in A. L. Koch, J. A. Robinson, G. A. Milliken (eds.), Mathematical Modelling in Microbial Ecology, Springer, Boston, 1998

[11] W. O. Kermack, A. G. McKendrick, Proc. R. Soc. Lond. A 115 (1927) 700-721.

[12] J. D. Murray, Mathematical Biology, $2^{\text {nd }}$ ed., Springer-Verlag, Berlin, 1993.

[13] D. J. Daley, J. Gani, Epidemic Modelling: An Introduction, Cambridge University Press, Cambridge, 1999.

[14] F. Brauer, C. Castillo-Chavez, Z. Feng, Mathematical Models in Epidemiology, Springer, New York, 2019.

[15] A partir das eqs. 13 e 14, conclui-se imediatamente que $E=-d S / d t$ é a função impulso para os infetantes, que decaem como $f(t)=\exp (-t / \tau)$, eqs. 16 e 17, ou seja, $I=E \otimes f+I_{0} f$, em que $\otimes$ representa a convolução

\section{Mário Berberan e Santos}

Professor Catedrático de Química-Física, Materiais e Nanociências do Instituto Superior Técnico, Universidade de Lisboa. Foi presidente da Sociedade Portuguesa de Química em 2010-13. Chemistry Europe Fellow (classe de 2018/19). Presidente do Conselho Coorde- nador do Colégio de Química da Universidade de Lisboa. A sua investigação incide principalmente sobre luminescência, incluindo processos de relaxação e aplicações como sensores e OLEDs. berberan@tecnico.ulisboa.pt ORCID.org/0000-0002-2946-1498. entre duas funções. Em condições supercríticas $\left(R_{0}>1\right)$, e sendo $I_{0}<<S_{0}, 0$ segundo termo é desprezável.

[16] P. van den Driessche, Infect. Dis. Model. 2 (2017) 288-303.

[17] A. J. Kucharski, T. W. Russell, C. Diamond, Y. Liu, J. Edmunds, S. Funk, R. M. Eggo, Lancet Infect. Dis. 20 (2020) 553-558.

[18] Report of the WHO-China Joint Mission on Coronavirus Disease 2019 (COVID-19), WHO, 28 Feb. 2020. Neste relatório indicam-se 2 semanas como tempo médio de recuperação para os casos leves e de 3-6 semanas para os casos graves.

[19] M. N. Berberan-Santos, submetido para publicação.

[20] News and Views, Brit. Med. J. 1 (6112) (1978) 587.

[21] M. Rozo, G. K. Gronvall, mBio 6 (2015) e01013-15.

[22] https://www.ecdc.europa.eu/en/geographical-distribution-2019-ncov-cases

[23] https://www.worldometers.info/coronavirus/coronavirus-death-rate/

[24] Mantendo a analogia com uma reação influenciada pela difusão, a constante de velocidade do controlo por difusão é $k_{d}=8 R T / 3 \eta$, sendo $R$ constante dos gases perfeitos, $T$ a temperatura e $\eta$ a viscosidade, que também varia com $T$, em primeira aproximação de acordo com a equação de Andrade, $\eta=A \exp \left(E_{2} / R T\right)$. Mas as medidas de contenção também afetam $p$, por exemplo pelo uso de máscaras, distanciamento, etc.

[25] http://www.insa.min-saude.pt/category/areas-de-atuacao/epidemiologia/ covid-19-curva-epidemica-e-parametros-de-transmissibilidade/

[26] H. E. Randolph, L. B. Barreiro, Immunity 52 (2020) 737-741.

[27] T. Britton, F. Ball, P. Trapman, Science 10.1126/science.abc6810 (2020).

[28] Veja-se, por exemplo, um estudo recente em que se utiliza um modelo SEIR para analisar vários cenários de desconfinamento: T. Rawson, T. Brewer, D. Veltcheva, C. Huntingford, M. B. Bonsall, Front. Public Health 8 (2020) 262. 